\title{
Diagrams: A Perspective from Logic
}

\author{
Dave Barker-Plummer \\ CSLI, Stanford University \\ dbp@stanford.edu
}

\section{Tutorial Overview}

The major goals of this two-hour tutorial are to to give an overview of this formal perspective on diagrams, and to introduce and explain the techniques used by logicians to analyze reasoning with diagrammatic representations.

The tutorial will describe the questions asked by logicians when analyzing the properties of diagrammatic representations and the techniques used to reason with them. The three main questions concern: expressive completeness - the degree to which diagrams can be used to represent information about a given domain; soundness of inference - how we can guarantee the validity of conclusions reached by reasoning with diagrams, and completeness of inference - the range of conclusions that can be reached by using those techniques.

The tutorial will proceed by first recapitulating the standard sentence-based approach to modelling reasoning, outline how these techniques can be applied to diagrammatic system, and then give a detailed presentation of the Hyperproof reasoning system. Hyperproof is a formal reasoning system, implemented as a computer application, for heterogeneous reasoning with diagrams and sentences. The Hyperproof system demonstrates that techniques from logic can be used to model very naturally a large class of everyday reasoning problems.

While Hyperproof will form the unifying frame of the tutorial, work by many colleagues in the field of diagrammatic reasoning will be discussed. These will be used to bring out both differences and similarities between approaches within the field. Hyperproof has been chosen since the diagrams that the program uses are related in an intuitive way to the situations that the diagrams represent. The Hyperproof system has this in common with everyday diagrammatic representations such as maps and schematics of machinery, for example. The demonstrated techniques are therefore more accessible when presented using Hyperproof than within other diagrammatic systems used to represent more abstract domains.

The tutorial will close with a discussion of a range of formal diagrammatic representations, outlining the ways in which more abstract representations are similar to Hyperproof, more appropriate for their own domains and often more straightforward to analyze formally. We will draw examples inter alia from mathematics, in the realm of set theory using Venn diagrams, and puzzle diagrams such as sudoku. 